ISAHP 1996, Vancouver, Canada, July 12-15, 1996

\title{
SOME NEW RESULTS ON PRIORITY VECTORS EVALUATION: METHODOLOGY AND NUMERICAL MODELLING
}

\author{
Stan Lipovetsky \\ 1451 Overing Str., \#19-D, Bronx, NY 10461, USA \\ 102170.2625@compuserve.com
}

\begin{abstract}
Several approaches are elaborated for simultaneous evaluation of both right and left AHP priority vectors by transforming pairwise ratio matrices of the Saaty kind into skew-symmetric matrices of normalized pairwise differences. A complex matrix of pairwise ratios is also introduced. It is shown that the principal complex eigenvector of skewsymmetric and of complex matrices presents by its real and imaginary parts the mutual priority-antipriority estimates of preferability of items. A geometric representation of combined measures of preferability is applied by projecting priority-antipriority values of items onto the curve of the rectangular hyperbola of their relationship. Numerical modelling is performed for combined priority vectors. Additionally, generalized mean forms are used for constructing priority vectors in optimizing procedures. Numerical results indicate that the optimal solution could be obtained with the generalized means corresponding to the evaluation of priority vectors as principal eigenvectors, and not to the multiplicative kind of estimation.
\end{abstract}

\section{Introduction.}

In this study we concentrate on one of the methodological problems of the AHP, namely the connection between the left and right eigenvectors of a pairwise comparison matrix. According to conventional usage, the right principal eigenvector serves as an estimator for priorities among objects under consideration, and the left principal eigenvector gives the reciprocal values of the same priorities (Saaty, 1980, 1994a). The importance of the left eigenvector was noticed in (Johnson, Beine and Wang, 1979) where in addition to a right vector indicating the "quality" of objects, a left eigenvector was defined as a "disquality" index. These authors noted that the two vectors generated different sets of preference values and they suggested taking the arithmetic mean of the corresponding elements of the right and the elementwise reciprocal left eigenvectors to produce a final ranking vector. This problem of the inverse inconsistency of the right and left vectors is considered with many interesting examples in (Dodd, Donegan and McMaster, 1995).It may, in fact, be that the scale of "goodness" (or "preferability") might not coincide with the scale of "badness" (or "non-preferability"). These scales might be more or less anticolinear, but not be exactly opposite. If the scales defined by the right and the left eigenvectors are indeed oriented differently, they are able to produce different ranking orders. This applies particularly to intermediate items, whereas the "best" and "worst" objects are usually located at the extreme positions of both scales.

The methods presented in this work simultaneously consider both the left and the right priority vectors by transforming reciprocally symmetric pairwise ratio matrices of the Saaty kind into skew-symmetric matrices of normalized pairwise differences. Skew-symmetric matrices (matrices with elements $a_{\mathrm{ij}}=a_{\mathrm{ji}}$ ) have been used in multidimensional scaling and taxonomy analysis based on principal component analysis (Gower, 1977; Constantine and Gower, 1978; see also Gabriel, 1971; Escoufier and Grorud, 1980; Chino, 1990). In the AHP environment, skew-symmetric matrices have been applied in (Genest, Lapointe and Drury, 1993; Genest and Zhang, 1994) in order to graphically represent consistency measures in pairwise comparisons. Vargas (1994) compared the AHP with multi-attribute utility theory (MAUT), and Fishburn (1984, 1988, 1991) developed the concept of skew-symmetric bilinear (SSB) forms for multiattribute utility functions; their structure corresponds to the antisymmetricized outer product of two vectors (see also Bell and Farquhar, 1986; Lavalle and Fishburn, 1987). Fishburn (1984) indicates a natural property of such SSB forms - the possibility of preference reversals.

In the present work we operate with complex eigenvectors of skew-symmetric matrices and of complex matrices. Complex eigenvectors were considered in principal component analysis in (Gupta, 1972; see also Goodman, 1963; Khatri, 1965). In the AHP complex matrices and eigenvectors were used in (Lambert, 1991). 
Consider the classic AHP. The most important inputs into priority weights are given by the elements $a_{i j}>1$ of a pairwise ratios matrix. Each new iteration in vector assessment corresponds to a linear combination of the previous estimation $a_{i}$ with weights of the elements in each row of the matrix $A$. Thus, the influence of values $a_{i j}>1$ and $a_{i j}<1$ of a reciprocally symmetric matrix $A$ is quite unsymmetric in the evaluation of the estimators $a$ and $b$ for preferences and non-preferences of the items.

In the multiplicative mode of the AHP priority weights $a_{i}$ are proportional to the geometric means of the elements in rows of a matrix $A$. In the same way, the dual vector of reciprocal weights can be estimated by vector $\mathrm{b}$ of geometric means of the elements in the columns of a matrix. This method is a well-known approach, used in the AHP (Saaty and Vargas, 1984; Saaty and Kearns, 1985; Golden, Wasil and Harker, 1989; Lootsma, 1993). We refer to this approach as a logarithmic method (LN).

\section{Skew-symmetrization in the AHP}

The LN method corresponds to evaluating a priority vector by a transformed pairwise comparison matrix, where all the elements $a_{i j}$ in matrix and priority weights are changed to their logarithms. This is a skewsymmetric matrix, i.e., in such a matrix the significance of the symmetric elements is equal. Theil (1971) suggested using logarithms of economic indices instead of the indices themselves, in order to equalize rates and their inverse values.

Using logarithms of priorities we can consider a generalization of the LN solution. Relations between priorities and their ratios could be expressed in a more general form as products of power functions (see De Jong, 1967, for construction of such relations by the principles of dimensional analysis; Saaty, 1994b, for power functions in priority compositions). This generalized logarithmic (GLN) approach can be reduced to an eigenproblem of a matrix of logarithms of pairwise ratios. The first eigenvector defines the weights $\mathrm{g}_{\mathrm{j}}$ ,and priorities are proportional to weighted geometric means of elements in rows of a Saaty matrix. In the case of all equal constants $g_{j}$, the GLN relations are reduced to the expressions of the LN model. Then LN solution corresponds to the first approximation in the iterative process of calculation for $\mathrm{g}$ in the GLN eigenproblem.

A question arises: what do they mean, the elements of a transformed matrix of logarithms of a Saaty matrix elements? In other words, how to interpret a logarithm of the ratio of two priorities and a skew-symmetric matrix of all of these pairs? To answer this question, let us introduce a so-called logarithmic mean $\mathrm{Lij}$ of two positive numbers, well known in the Index Analysis (see Vartia, 1976; Sato, 1976; Carlson, 1972; Tornquist, Vartia and Vartia, 1985). Lij falls in the range between the geometric and arithmetic means. This logarithmic mean is also called a chronologic mean, and could be obtained as a harmonic mean of a uniformly distributed continuous variable (Lipovetsky, 1983).

Now we can easily interpret a skew-symmetric matrix with elements equal to logarithms of Saaty pairwise ratios: elements of the transformed matrix are pairwise differences of priorities, normalized by their mean values. This property open a possibility to fulfill a systematic comparison between solutions (preferability vectors) that could be produced by methods operating with relative and absolute differences of priorities.

Using another mean value between priorities corresponds to another kind of transformation of a matrix A to a skew-symmetric form. Consider a geometric mean, then for the elements of a transformed matrix we can take square roots of the elements of a Saaty matrix, arrange with them another matrix B, and construct a skew-symmetric matrix by subtraction of $B$ transposed from $B$. Elements of a skew-symmetric matrix could be constructed with other normalizations too. For example, with a harmonic mean the transformation of a pairwise reciprocally symmetric Saaty matrix into a skew-symmetric matrix of normalized pairwise differences could be presented as half of the difference between A and A transposed. For a skew-symmetric matrix of each kind an eigenproblem can be solved, and the principal eigenvector may then be transformed into a priority vector.

Let us consider the properties of the eigenproblem solution for a skew-symmetric matrix. As is known in linear algebra (Guntmacher, 1959, 1960), a skew-symmetric matrix has an even number of purely imaginary eigenvalues, that are self-conjugate pairs, and other eigenvalues equal zero. Eigenvectors of a skewsymmetric matrix are complex conjugate vectors $z$ corresponding to these eigenvalues. It can be shown that the first pair of eigenvectors $z 1, z 2$ (associated with the maximal by the absolute value eigenvalue) define by their real and imaginary parts two real vectors $a$ and $b$ which serve as estimators for two dual priority vectors. (More precisely, from these pre-priority vectors, we obtain the estimators $a$ and $b$ of the theoretical priority vectors $w$ and $v$ ). The estimator $a$ is a vector of preferability weights among items under 
consideration, and $b$ serves as an estimator of their component-wise inverted values. Thus, we will refer to $a$ as the priority vector, and to $b$ as the antipriority vector.

We also consider another possibility of the simultaneous estimation of priority-antipriority vectors of a complex matrix $S=A+i A^{\prime}$. Here $A$ is a Saaty matrix used in the AHP eigenproblem for the right vector, and $A^{\prime}$ is a transposed matrix used in the AHP eigenproblem for the left vector. In this matrix $S$ the real part corresponds to priority estimation by the matrix $A_{1}$ and the imaginary part is related to antipriority estimation by the matrix $A^{\prime}$. The eigenproblem for this matrix yields (see Lipovetsky, 1995) a pair of principal eigenvectors. The real and imaginary parts $a$ and $b$ of these vectors define two estimators of priority-antipriority vectors. Due to the symmetry of matrix $S$, the solutions of these eigenproblems do not change, if in place of this matrix we take a transposed matrix $A^{\prime}+i A$.

\section{Numerical comparisons with dual vectors}

Numerical experiments shows that different methods produce pairs of vectors that may vary in weights and in rank ordering. By most estimators, the ranks are the same only for the best item and for the worst item. For intermediate objects, the vectors $a$ and $b$ give a different ranking to the same object even within the same method of estimating. Only the LN method produces the same results for vector a and its corresponding elementwise inverted vector $b$, but as noted in (Dodd, Donegan and McMaster, 1995) this does not mean much because this could be true for highly inconsistent matrices as well. The methods that use skew-symmetric matrices yield results similar to the better known AHP, Least Squares, and LN approaches, and show the expected rank ordering of all items.

Additionally, generalized means forms were used for approximation of the elements of pairwise priority matrices. Several optimizing criteria were used for this approximation (see Lipovetsky, 1996). The results shows that for the matrices of order more than three the best approximation could be found with the parameter of generalized means closed to one, and not to zero. It corresponds to the conclusion that optimal solutions are related to methods of eigenvector estimation of priority vectors, and not to the multiplicative mode ( $\mathrm{LN}$ solution corresponds to the generalized means when the parameter value is close to zero).

Concerning the combined estimation of rank ordering by both priority and antipriority vectors together, we suggest considering their elements as points on the complex plane ( $x$, iy). All.items can be ordered by the value of the angle between the real axis $x$ and vectors spreading from the origin of coordinates to the pair of points (ai, bi). Another way of ordering items by both priority-antipriority vectors can be obtained as the projection of points $(\mathrm{ai} ; \mathrm{bi})$ along the direction of the complex vectors to the hyperbolic curve, because for theoretical vectors of priority a hyperbola is the locus of weights $w_{\mathrm{i}}$ and $v_{i}=1 / w_{\mathrm{j}}$. The point of intersection of such ray with this curve can be expressed for each item as the geometric mean with a pair of priority and inverted antipriority values.

\section{Concluding Remarks}

This paper describes some problems of the dual estimation of priority vectors as left-right pairs of eigenvectors for a matrix of pairwise ratios and its transformed versions. In addition, to the more conventional methods of estimation in the AHP, we introduce several new approaches based on the evaluation of priority-antipriority vectors by skew-symmetric matrices and by complex matrices. The simultaneous consideration of both vectors as real and imaginary parts of a structurally more complicated complex vector could be useful in description and evaluation in the AHP setting of priority.

Difference in preferences of items, estimated by scales of preferability and non-preferability in parallel, could be checked using appropriate psychological measurements. It could also be interesting to investigate in psychological experiments the procedure of constructing matrix A. It seems intuitively more sound if a judge were to determine the elements not in the upper, but in the lower triangle of matrix A. This means that $\mathrm{s} /$ he gives the values of indices with a constant base (ratio of all preferences $w_{i}$ to one of them for each $\mathrm{j}$-th column of $\mathrm{A}$ ), and not the values of indices with changing base (ratio of the preference $w_{\mathrm{j}}$ to each of them in rows of $A$, as it is in the classic AHP). Following this approach, a judge fills the lower triangle of a sample matrix, and the upper triangle can be obtained by the reciprocal relation. Estimating of indexes with a fixed base is usually more consistent because it corresponds to a linear scale transformation (division by a constant for every column), whereas the classic AHP methodology uses a nonlinear scale transformation with division of the constant by different values of elements in denominators of ratios in rows A. It is also possible to obtain the upper and lower triangles of $A$ independently. Of course, elements of such a matrix would be more inconsistent, but this inconsistency in expert estimation has another side - more reliable 
results in statistical evaluation, because in this case, the elements of vectors a and $b$ could be estimated by twice the number of independent elements above and below the diagonal of matrix $A$ of pairwise comparisons. All the methods for priority estimations could be redefined for such non-symmetric (even in the reciprocal sense) matrices.

Some other remarks concern the possibility of considering the mutual priority-antipriority ordering of items as a cooperative game of a duopolistic kind (Henderson and Quandt, 1980; Moulin, 1988; Myerson, 1991). In priority-antipriority duopoly, the ranking of items may be characterized by a combination of competitive and cooperative aspects of the ordering. Each item occupies its particular position in a two-dimensional space of two attributes determined by levels of priority and antipriority. Maximizing utility corresponds to elaborating the best rank ordering and could be considered according to the Nash (1950) bargaining solution. For duopoly, this solution is defined as a locus of utility points lying on the curves of rectangular hyperbolas. The competitive market of many items described by two attributes corresponds to balancing for priority-antipriority ranking by projecting the items on the hyperbolic curve of their connection.

Under the assumption that different pairs of priority-antipriority weights could be reciprocaliy connected but with different constants of their connection for each $i$, we have a family of hyperbolas with different values for the parameter, corresponding to changing utility levels of the equilibrium of dual attributes. Such a more general description of the priority-antipriority connection can be expressed as a dependence $f=x y$ where $x$ and $y$ are independent variables of priority and antipriority, and $f$ is a function that presents levels of the utility (preferability) of each itern. The last expression describes the saddle-shaped surface of a hyperbolic paraboloid, well known in analytic geometry.

The concept of hyperbolic geometry was initiated in the AHP in another context by Saaty (1994c). Properties of this surface useful for index analysis were considered in (Lipovetsky, 1983). For now, we note that the variables $x$ and $y$ correspond to the estimates $a$ and $b$, and positioning of items (ai, bi) on the surface $f$ determines a curve of priority locus in 3-dimensional space. Thus, considering this curve's behavior could help in elaborating adequate characteristics for preference ordering. On the other hand, all the combinations in products of ai and bj for the items under consideration $(i, j=1, \ldots, n)$ correspond to estimations of ratios wi/wj. Therefore all the elements of a Saaty matrix can be considered as points on the surface of a hyperbolic paraboloid with deviations from their locus because of inconsistent estimations of pairwise ratios. This means that to elaborate the inconsistency characteristics, we can use properties of the geometric representation of pairwise ratios.

This work extends the AHP into direction of complex matrices of pairwise comparisons and complex vectors of dual priority-antipriority ordering of items simultaneously in ascending and descending directions of preferability. This capability could be useful for theoretical descriptions as well as for practical purposes of multiple criteria decision making problems.

\section{References}

Bell D.E. and P.H. Farquhar (1986), "Perspectives on Utility Theory", Operations Research, 34, No. 1, 179-183.

Carison B. (1972), "The Logarithmic Mean", American Mathematical Monthly, 79, 615-618.

Chino N. (1990), "A Generalized Inner Product Model for the Anaiysis of Asymmetry", Behaviormetrika, $27,25-46$.

Constantine A.G. and J.C. Gower (1978), "Graphical Representation of Asymmetric Matrices", Applied Statistics, 27, No. 3, 297-304.

De Jong F.J. (1967), Dimensional Analysis for Economists. North-Holland Publishing Company, Amsterdam.

Dodd F.J., H.A. Donegan and T.B.M. McMaster (1995), "Inverse Inconsistency in Analytic Hierarchies", European Journal of Operational Research, 80, No. 1, 86-93.

Escoufier Y., Grorud A. (1980), "Analyse Factorielle des Matrices Carrées Non Symetriques" - in Diday E. et al. (Eds.), Data Analysis and Informatics, 263-276, Amsterdam, North Holland.

Fishburn P.C. (1984), "Dominance in SSB Utility Theory", Journal of Economic Theory, 34, No. 1, 130148.

Fishburn P.C. (1988), Nonlinear Preference and Utility Theory. Johns Hopkins University Press, Baltimore. 
Fishburn P.C. (1991), "Nontransitive Preferences in Decision Theory", Journal of Risk and Uncertainty, 4, No. 2, 113-134.

Gabriel K.R. (1971), "The Biplot Graphic Display of Matrices with Application to Principal Component Analysis", Biometrika, 58, No. 3, 453-467.

Genest C., F. Lapointe and S.W. Drury (1993), "On a Proposal of Jensen for the Analysis of Ordinal Pairwise Preferences Using Saaty's Eigenvector Scaling Method", Journal of Mathematical Psychology, 37, No. 4, 575-610.

Genest C. and S.S. Zhang (1994), "The Graphical Analysis of Ratio-Scaled Paired Comparison Data", Prepublications, Department de Mathematiques et de Statistique, Universite Laval, Quebec.

Golden B.L., E.A. Wasil and P.T. Harker (eds.) (1989), The Analytic Hierarchy Process: Applications and Studies. Springer-Verlag, Heidelberg.

Goodman N.R. (1963), "Statistical Analysis Based on a Certain Multivariate Gaussian Distribution", Annals of Mathematical Statistics, 34, 152-177.

Gower J.C. (1977), "The Analysis of Asymmetry and Orthogonality", in: Barra J.R. et al. (Eds.), Recent Developments in Statistics, 109-123, Amsterdam, North Holland.

Guntmacher F.R. (1959), Applications of the Theory of Matrices. Interscience Publ. Inc., NY.

Guntmacher F.R. (1960), The Theory of Matrices. Chelsea Publishing Company, NY.

Gupta R.P. (1972), "Principal Component Analysis in the Complex Case". Metrika, 19, No. 2-3, 150-155.

Henderson J.M. and R.E. Quandt (1980), Microeconomic Theory: A Mathematical Approach. McGraw Hill, Auckland.

Johnson C.R., W.B. Beine and T.J. Wang (1979), "A Note on Right-Left Asymmetry in an Eigenvector Ranking Procedure", Journal of Mathematical Psychology, 19, No. 1, 61-64.

Khatri C.G. (1965), "Classical Statistical Analysis Based on a Certain Multivariate Complex Gaussian Distribution", Annals of Mathematical Statistics, 36, 98-114.

Lambert J. M. (1991), "The Extended Analytic Hierarchy Decision Method", Mathematical and Computer Modelling, 15, No. 11, 141-151.

Lavalle I.H. and P.C. Fishburn (1987), "Decision Analysis under States-Additive SSB Preferences", Operations Research, 35, No. 5, 722-735.

Lipovetsky S. (1983), "Variational Analysis of the Breakdown of an Increase between Factors", Matekon, XX, No. 1, 93-103, M.E. Sharp Inc., NY.

Lipovetsky S. (1995), "Dual Priorities in the AHP: Complex Solutions", Faculty of Management, Tel"Aviv University, Working Paper No. 16/95.

Lipovetsky, S. (1996), "The Synthetic Hierarchy Method: An Optimizing Approach to Obtaining Priorities in the AHP", Forthcoming in European Joumal of Operational Research.

Lootsma F.A. (1993), "Scale Sensitivity in the Multiplicative AHP and SMART", Journal of Multi-Criteria Decision Analysis, 2, No. 2, 87-110.

Moulin M. (1988), Axioms of Cooperative Decision Making. Cambridge: Cambridge University Press.

Myerson R.B. (1991), Game Theory: Analysis of Conflict. Cambridge: Harvard University Press.

Nash J.F. (1950), "Equilibrium Points in n-Person Games", Proceedings of the National Academy of Sciences, USA, 36, 48-49.

Saaty T.L. (1980), The Analytic Hierarchy Process. N.Y.: McGraw-Hill.

Saaty T.L. (1994a), Fundamentals of Decision Making and Priority Theory with the Analytic Hierarchy Process. RWS Publications, Pittsburgh.

Saaty T.L. (1994b), "Observations on Multiplicative Composition in the Analytic Hierarchy Process", Proceedings of the 3rd International Symposium on the Analytic Hierarchy Process, Washington, DC, USA, 169-176. 
Saaty T.L. (1994c), "Reciprocal Comparisons, Inversion and Hyperbolic Geometry: from Psychology to Geometry", Proceedings of the 3rd International Symposium on the Analytic Hierarchy Process, Washington, DC, USA, 41-60.

Saaty T.L. and K.P. Kearns (1985), Analytical Planning. Oxford: Pergamon Press.

Saaty T.L. and L.G. Vargas (1984), "Comparisons of Eigenvalue, Logarithmic Least Squares and Least Squares Methods in Estimating Ratios", Mathematical Modeling, 5, No. 5,309-324.

Sato K. (1976), "The Ideal Log-Change Index Number", The Review of Economics and Statistics, 58, No. 2, 223-228.

Theil, M. (1971), Principles of Econometrics. John Wiley \& Sons, NY, London.

Tornquist L., Vartia P. and Y. Vartia (1985), "How Should Relative Changes be Measured?", The American Statistican, 39, No. 1, 43-46.

Vartia Y.O. (1976), "Ideal Log-Change Index Numbers", Scandinavian Journal of Statistics, 3, No. 3, 121-126.

Vargas L. (1994), "Comparison of Three Muiticriteria Decision Making Theories: The Analytic Hierarchy Process, Multiattribute Utility Theory and Outranking", Proceedings of the 3rd International Symposium on the Analytic Hierarchy Process, Washington, DC, USA,19-29. 